\title{
INFLUENCE OF MOMENTUM-DEPENDENT INTERACTIONS ON THE NUCLEAR STOPPING IN SYMMETRIC HEAVY-ION COLLISIONS
}

\author{
MANDEEP KAUR, SUNEEL KUMAR
}

PACS 25.70.Pq, 25.70.-z,

School of Physics and Materials Science, Thapar University

(C) 2012

(Patiala-147004, Punjab (India); e-mail: suneel. kumar@ thapar. edu)

\begin{abstract}
The role of momentum-dependent interactions (MDI) in the nuclear stopping at intermediate energies using an isospin-dependent quantum molecular dynamics model is studied. The calculations are performed for incident energies between 50 and $1000 \mathrm{MeV} /$ nucleon. Our findings show that the nuclear stopping is sensitive to the impact parameter, incident energy, and the mass of the colliding system. The degree of stopping is suppressed by the inclusion of MDI, whereas the particle production is not affected by MDI.
\end{abstract}

\section{Introduction}

The study of heavy ion collisions (HIC) at intermediate energies has been of central interest due to the fact that this gives us a possibility to understand the behavior of hot and dense nuclear matter under the extreme conditions of temperature, pressure, densities, and collective effects appearing in such systems, differing from particle physics, where one studies the interactions between the elementary particles [1-4]. The research in last few years was focused mainly on the origin of fragmentation, also known as multifragmentation [1,2], as well as on the movement of particles along different directions, i.e., a collective directed flow and an elliptic flow [5]. Huge experimental data for the multifragmentation helps to answer the fundamental questions like the origin of fragments, mechanism behind the fragmentation, and the role of dynamical correlations in their formation [6-9].

The nuclear stopping which is also related to the above-listed various mechanisms happening at intermediate-energy HIC. It is the transformation of nuclear matter from a fused state to the total disassembly and vaporization. The complete destruction of nuclear matter is termed as the global stopping. The nuclear stopping can be divided into zones of full stopping, partial one, and the transparency of the colliding pair. A full stopping means the whole of the projectile nucleons are stopped by target nucleons and vice versa. The partial stopping means that some of the nucleons are stopped, while the rest of the nucleons are allowed to cross. Lastly, the transparency of nucleons denotes the crossing of whole of the projectile nucleons toward the target and vice versa. The amount of nuclear stopping determines parameters, such as the energy and the volume of the interaction region, which govern the reaction dynamics and the extent, to which conditions might be favorable for the formation of a highly dense, de-confined phase matter. At intermediate energies, the mixture of the attractive mean field and the repulsive nucleonnucleon scattering makes this process much more complicated. The degree of stopping also depends on the incident energy, mass of colliding nuclei, and geometry of the system. Here, we consider few quantities capable of estimating the degree of global stopping. We also try to correlate it with the production of light charged particles originating from the participant hot dense zone. One has tried to correlate the light charged particles (LCPs) with nuclear stopping parameters in HIC using the quantum molecular dynamics (QMD) and isospindependent quantum molecular dynamics (IQMD) models. In nucleus-nucleus collisions, the stopping can be seen as a shift of the rapidity distribution of the incident nucleons toward the mid-rapidity region. Thus, the shape of rapidity distributions provides a key information in terms of the nuclear stopping power. Bauer et al. pointed out that, in intermediate-energy HIC, 
the nuclear stopping is determined by both the mean field and the in-medium nucleon-nucleon cross-section [9]. They, however, neglected the symmetry potential in their calculations. Later, Bass, Yennello, Johnston, and co-workers suggested that the degree of approaching the isospin equilibration provides a means to probe the mechanism and the power of nuclear stopping in heavyion collisions [10].

At the same time, it is seen that the momentumdependent interactions (MDI) play a very important role in determining the nuclear dynamics [10]. It is an important feature for the fundamental understanding of the nuclear matter properties over a wide range of densities and temperatures. The initial attempts with MDI have showed a drastic effect on the collective flow, as well as the particle production (i.e., it results in a large number of fragments at peripheral collisions) [11, 12]. The effect of MDI is strongest during the initial phase of the reaction. The particles propagating with MDI are accelerated in the transverse direction during the early phase of the reaction. As a result, fewer collisions take place, and the transverse flow increases considerably. Our main motive is to study the nuclear stopping by taking the momentum dependence of the reaction into account. The present work is done within the IQMD model described in the next section.

\section{The Model}

The IQMD [13] model treats different charge states of nucleons, deltas, and pions explicitly, as inherited from the Vlasov-Uehling-Uhlenbeck (VUU) model. The IQMD model has been used successfully in analyzing the large number of observables from low to relativistic energies. The isospin degree of freedom enters into the calculations via both cross sections and the mean field. In this model, baryons are represented by Gaussian-shaped density distributions

$f_{i}(r, p, t)=\frac{1}{\pi^{2} \hbar^{2}} e^{\frac{-\left(r-r_{i}(t)\right)^{2}}{2 L}} e^{\frac{-\left(p-p_{i}(t)\right)^{2} \cdot 2 L}{\hbar^{2}}}$.

Nucleons are initialized in a sphere with the radius $R=1.12 A^{1 / 3} \mathrm{fm}$, in accordance with the liquid drop model. Each nucleon occupies a volume of $\hbar^{3}$, so that the phase space is uniformly filled. The initial momenta are randomly chosen between 0 and the Fermi momentum $p_{\mathrm{F}}$. The nucleons of the target and the projectile interact via two- and three-body Skyrme forces and the Yukawa potential. The isospin degrees of freedom are treated explicitly by employing a symmetry potential and explicit Coulomb forces between protons of the colliding target and the projectile. This helps in achieving the correct distribution of protons and neutrons in a nucleus. The hadrons propagate by the Hamilton equations of motion:

$\frac{d \mathbf{r}_{i}}{d t}=\frac{d\langle H\rangle}{d \mathbf{p}_{i}}, \quad \frac{d \mathbf{p}_{i}}{d t}=-\frac{d\langle H\rangle}{d \mathbf{r}_{i}}$

where $\langle H\rangle=\langle T\rangle+\langle V\rangle$ is the Hamiltonian

$\sum_{i} \frac{p_{i}^{2}}{2 m_{i}}+\sum_{i} \sum_{j>i} \int f_{i}(\mathbf{r}, \mathbf{p}, t) V^{i j}\left(\mathbf{r}^{\prime}, \mathbf{r}\right) f_{j}\left(\mathbf{r}^{\prime}, \mathbf{p}^{\prime}, t\right) \times$

$\times d \mathbf{r} d \mathbf{r}^{\prime} d \mathbf{p} d \mathbf{p}^{\prime}$

In the above relation, the baryon-baryon potential $V^{i j}$ reads

$V^{i j}\left(\mathbf{r}^{\prime}-\mathbf{r}\right)=V_{\text {Skyrme }}^{i j}+V_{\text {Yukawa }}^{i j}+V_{\text {Coul }}^{i j}+V_{\text {Sym }}^{i j}+V_{\text {mdi }}=$

$=t_{1} \delta\left(\mathbf{r}^{\prime}-\mathbf{r}\right)+t_{2} \delta\left(\mathbf{r}^{\prime}-\mathbf{r}\right) \rho^{\gamma-1}\left(\frac{\mathbf{r}^{\prime}+\mathbf{r}}{2}\right)+$

$+t_{3} \frac{\exp \left(\left|\mathbf{r}^{\prime}-\mathbf{r}\right| / \mu\right)}{\left(\left|\mathbf{r}^{\prime}-\mathbf{r}\right| / \mu\right)}+\frac{Z_{i} Z_{j} e^{2}}{\left|\mathbf{r}^{\prime}-\mathbf{r}\right|}+$

$+t_{4} \frac{1}{\rho_{o}} T_{3}^{i} T_{3}^{j} \delta\left(\mathbf{r}_{i}^{\prime}-\mathbf{r}_{j}\right)+t_{5} \ln ^{2}\left(1+t_{6}\left(\mathbf{p}^{\prime}-\mathbf{p}\right)^{2} \delta\left(r^{\prime}-\mathbf{r}\right)\right.$.

Here, $\mu=1.5 \mathrm{fm}, t_{3}=-6.66 \mathrm{MeV}, t_{4}=100 \mathrm{MeV}$. The values of $t_{1}$ and $t_{2}$ depend on the values of $\alpha, \beta$, and $\gamma$ [1]. Here, $Z_{i}$ and $Z_{j}$ denote the charges of the $i^{\text {th }}$ and $j^{\text {th }}$ baryon, and $T_{3}^{i}, T_{3}^{j}$ are their respective $T_{3}$ components (i.e., $1 / 2$ for protons and $-1 / 2$ for neutrons). The parameters $\mu$ and $t_{1}, \ldots, t_{6}$ are adjusted to the real part of the nucleonic optical potential.

\section{Results and Discussions}

Nuclear stopping in HIC has been studied with the help of different variables. A direct measure of nuclear stopping is the rapidity distribution defined as [14]

$Y(i)=\frac{1}{2} \ln \frac{E(i)+p_{\|}(i)}{E(i)-p_{\|}(i)}$,

where $E(i)$ and $p_{z}(i)$ are respectively, the energy and the longitudinal momentum of the $i^{\text {th }}$ particle. For a complete stopping, one expects a single peaked Gaussian. Obviously, a narrow Gaussian indicates the better thermalization (equilibrium) compared to broader ones. 
Another quantity used in the literature [14] is the anisotropy ratio $(R)$ defined as

$R=\frac{2}{\pi} \frac{\left[\sum_{i}\left|p_{\perp}(i)\right|\right]}{\left[\sum_{i}\left|p_{\|}(i)\right|\right]}$

where the summation runs over all nucleons. The transverse $p_{\perp}(i)$ and longitudnal $p_{\|}(i)$ momenta read, respectively, as $\sqrt{p_{x}^{2}(i)+p_{y}^{2}(i)}$ and $p_{z}(i)$. Naturally, for the complete stopping, $R$ should be close to 1 . Some studies use the quadrupole moment $Q_{z z}$ to analyze the stopping and the thermalization. Quadrupole moment $Q_{z z}$ is defined as

$Q_{z z}=\sum_{i}\left[2 p_{\|}^{2}(i)-p_{\perp}^{2}(i)\right]$

Naturally, for the complete stopping, $Q_{z z}$ should be close to zero.

In the present analysis, thousands of events were simulated for the neutron-rich reaction of ${ }_{54} \mathrm{Xe}^{131}+{ }_{54} \mathrm{Xe}^{131}$, at incident energies between 50 and $1000 \mathrm{MeV} /$ nucleon using the hard momentum dependent equation of state (HMDE) and $E_{\text {sym }}=32 \mathrm{MeV}$. In Fig. 1, we display the final phase space of a single event of ${ }_{54} \mathrm{Xe}^{131}+{ }_{54} \mathrm{Xe}^{131}$, at an incident energy of $400 \mathrm{MeV} /$ nucleon for hard momentum dependent interactions (HMD). The top, middle, and bottom panels are at $\hat{b}=0,0.3$, and 0.6 , respectively. Here, we display the phase space of free nucleons $[A=1]$, light charged particles (LCPs) $[(2 \leq A \leq 4)]$, and intermediate mass fragments (IMFs) $\left[\left(5 \leq A \leq A_{\text {tot }} / 6\right)\right]$. It is clear from the figure that the complete spherical distribution of particles is obtained at central collisions indicating the spreading of nucleons in all directions, which implies that the breaking of the initial correlation is maximal in this region. As a result, the randomization increases, and the stopping in the hot compressed nuclear matter occurs. However, the MDI have a sizable effect on the charged particle multiplicity.

It is found that, in comparison to the simple static equation of state, the MDI lead to a large number of fragments at peripheral collisions, whereas it yields fewer fragments in central collisions [7]. Since the free particles and LCPs originate from the mid-rapidity region, they are better suited for studying the degree of stopping reached in HIC, whereas the IMFs originated from the target or projectile region are treated as the residue of the spectator matter $[10,16,17]$.

Figure 2 displays the rapidity distribution $d N / d Y$ for the emission of free nucleons, as well as LCPs and IMFs. The rapidity distribution gives a large value with MDI.

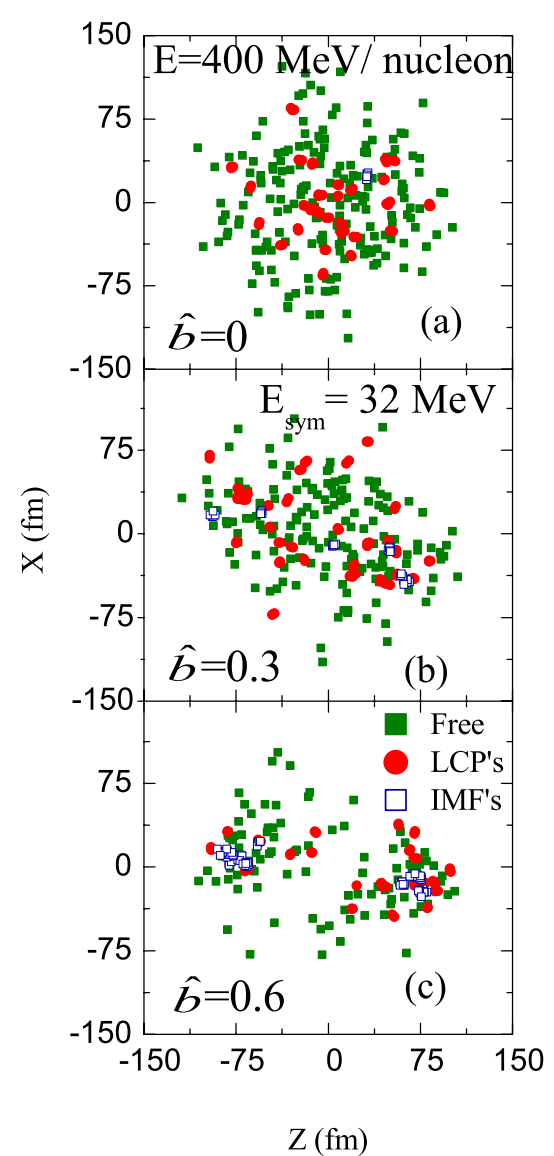

Fig. 1. Final phase space of a single event for the reaction of ${ }_{54} \mathrm{Xe}^{131}+{ }_{54} \mathrm{Xe}^{131}$ with a symmetry energy of $32 \mathrm{MeV}$. The top $(a)$, middle $(b)$, and bottom (c) panels are, respectively, for the scaled impact parameter $\hat{b}=0,0.3$, and 0.6. Different symbols are used for free nucleons, LCPs, and IMFs

The most striking point is that the rapidity is less affected in the central collisions, whereas it leads to entirely different results at peripheral collisions. This is due to fact that, in central collisions, the nucleonic collisions dominate the reaction, whereas the mean field governs the reactions in peripheral collisions. It is clear from the figure that free particles and LCPs emitted in the central collisions form a single narrow Gaussian shape, whereas IMFs have a broader Gaussian indicating the less thermalization. The single Gaussian distribution splits into two Gaussians (at target and projectile rapidities), as we shift toward peripheral collisions, indicating the correlated matter.

From the shape of the Gaussian, one sees that free particles and LCPs are better indicators of the thermalization [15]. At peripheral collisions, the spectator zone increases, and the participant zone becomes very small. 


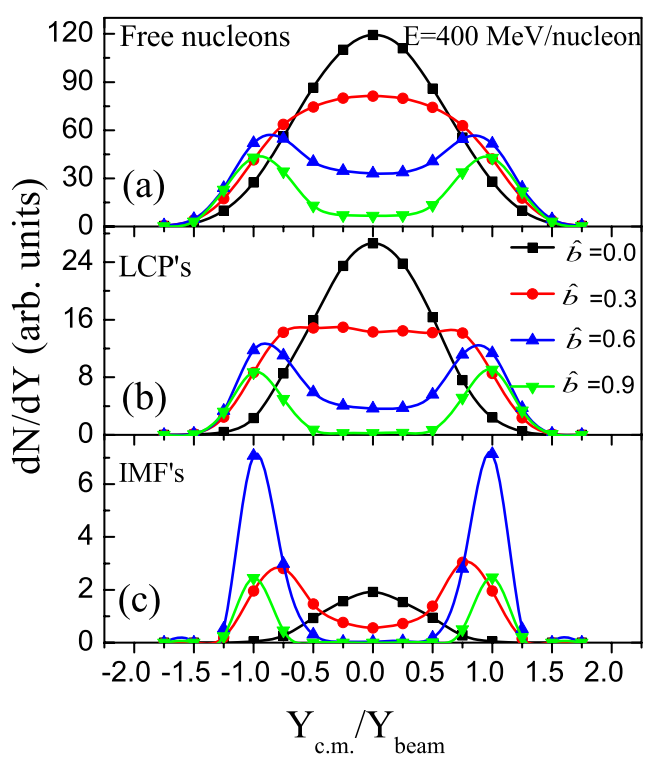

Fig. 2. Rapidity distribution $\mathrm{dN} / \mathrm{dY}$ as a function of the reduced rapidity for free nucleons $(a)$, LCPs $(b)$, and IMFs $(c)$ at different impact parameters, with symmetry energy. The reaction under study is ${ }_{54} \mathrm{Xe}^{131}+{ }_{54} \mathrm{Xe}^{131}$ at the incident energy $E=400$ $\mathrm{MeV} /$ nucleon

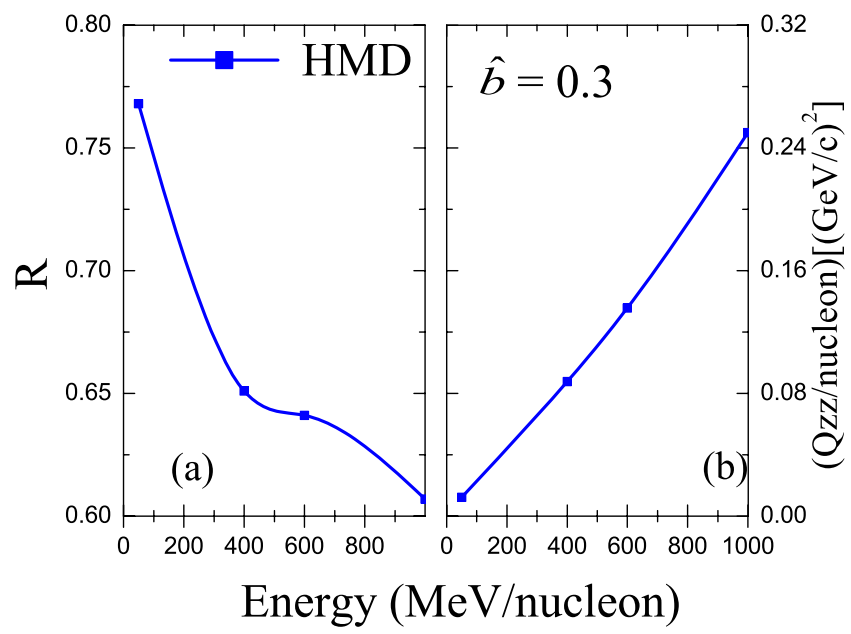

Fig. 3. Anisotropic ratio $R(a)$ and the quadrupole moment $Q_{z z}$ (b) as functions of the beam energy using HMDE

Most matter goes without any collision, and, hence, the rapidity of IMFs increases considerably.

In Fig. 3, we display the variation of the stopping parameters and the multiplicity of fragments with respect to the incident energy with hard momentum dependent equation of state. One can easily observe that the stopping decreases with increase in the incident energy. This is due to the increase in the transparency of

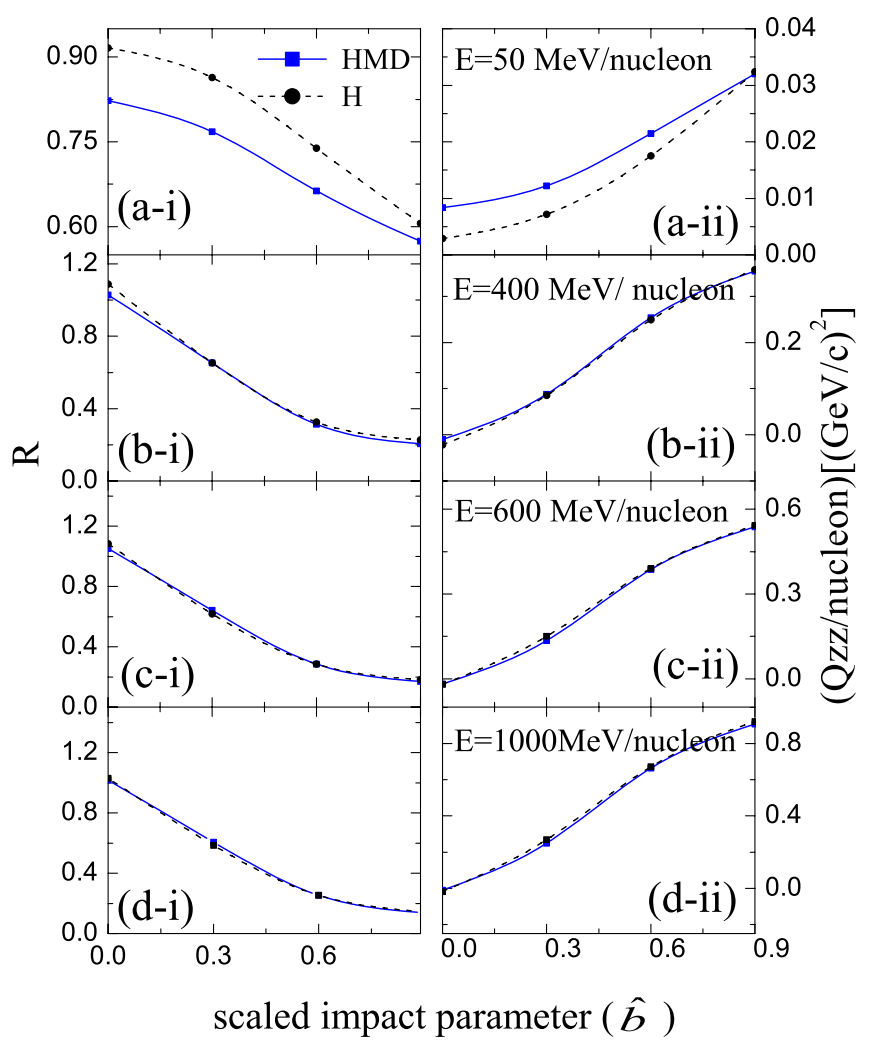

Fig. 4. Anisotropic ratio $R(i)$ and $Q_{z z} /$ nucleon $(i i)$ as functions of the scaled impact parameter with symmetry energy and MDI. The results obtained with HMDE and hard equation of state (HE), respectively, are represented by solid and dashed lines at the energies $50(a), 400(b), 600(c)$, and $1000 \mathrm{MeV} /$ nucleon $(d)$

colliding nuclei. At higher energies, the number of collisions decreases; therefore, $R$ approaches zero. At higher incident energies, the $z$ component of the momentum becomes stronger compared to the $x$ and $y$ components, and, hence, the value of $Q_{z z}$ also increases. The impact parameter dependence of the global variables $(R$ and $Q_{z z} /$ nucleon) has been displayed in Fig. 4. One can see that $R$ and $Q_{z z} /$ nucleon behave in opposite fashions. For $R>1$ and $Q_{z z} /$ nucleon $<0$, this can be explained by the preponderance of the momentum flow perpendicularly to the beam direction [16]. As the energy increases, $P_{z}$ becomes stronger, than $P_{x}$ and $P_{y}$.

So, the net value of $R$ decreases, indicating a less stopping. Whereas, with increase in the energy, the violence of $N-N$ collisions also increases. The maximum stopping is observed around $400 \mathrm{MeV} /$ nucleon, which is in supportive nature with the findings of Reisdorf et al. [17]. In their work, they measured the nuclear stopping for the energy range from 0.09 to 1.93 $\mathrm{GeV} /$ nucleon, and the maximal stopping was observed 


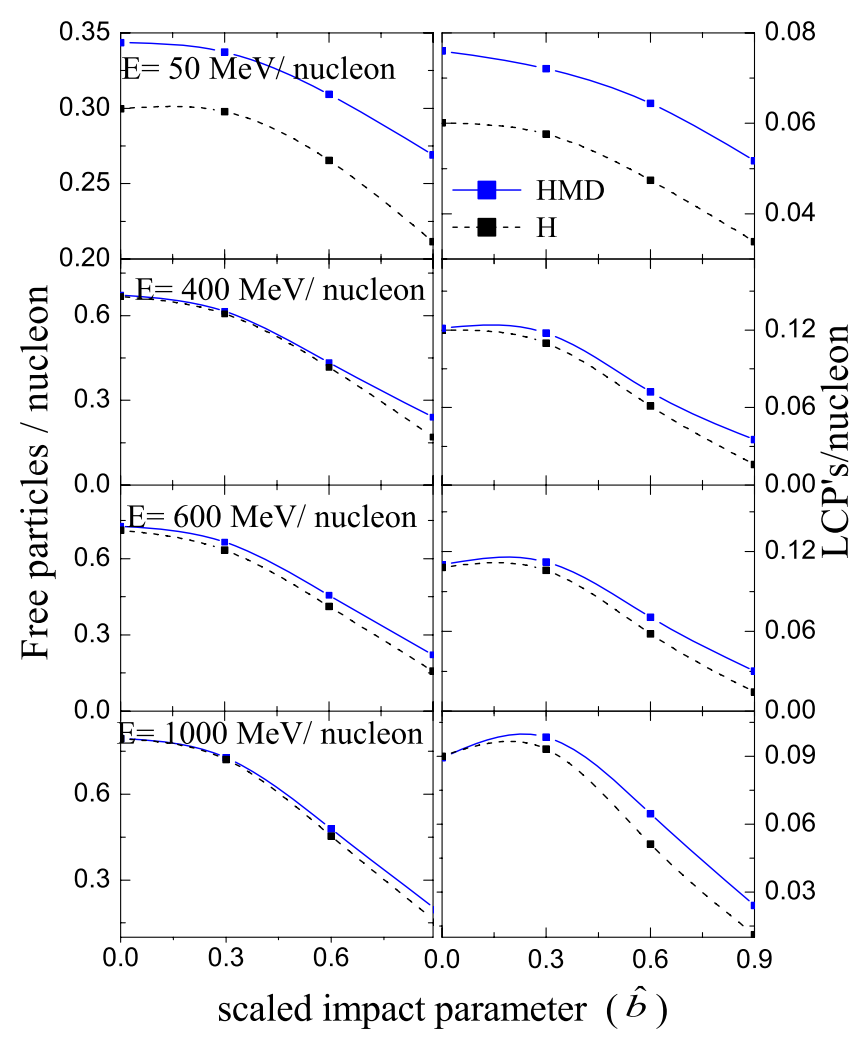

Fig. 5. Impact parameter dependence of the multiplicity of free particles and LCPs at energies equal to $50(a), 400(b), 600(c)$, and $1000 \mathrm{MeV} /$ nucleon $(d)$, respectively, with (solid line) and without momentum dependence (dotted line)

around $400 \mathrm{MeV} /$ nucleon. At a certain energy, when the reaction reaches a maximal stopping, the composite matter reaches a stage of the least transparency, and, thus, most of the particles are preferentially out of the plane. The effect of momentum dependent interactions is also visible in the nuclear stopping at lower incident energies, which decreases with the incident energy. At the high incident energy (1000 MeV/nucleon), the anisotropic ratio $R$ is independent of the equation of state (EOS). At low values of incident energy (50 MeV/nucleon), the anisotropic ratio with HMDE is less compared to that with the simple static EOS. This indicates that MDI suppress the nuclear stopping [10]. In Fig. 5, we display the impact parameter dependence of the multiplicity of free nucleons and LCPs at $200 \mathrm{fm} / \mathrm{c}$. The behavior of all the curves is in analogy with the nuclear stopping parameters $R$ and $1 /\left(Q_{z z} /\right.$ nucleon $)$, as discussed in Fig. 4. We see that the multiplicity of free nucleons decreases with increase in the impact parameter due to a decrease in the participant region. As a result, LCPs also decrease with increase in the impact parameter. The effect of MDI de-

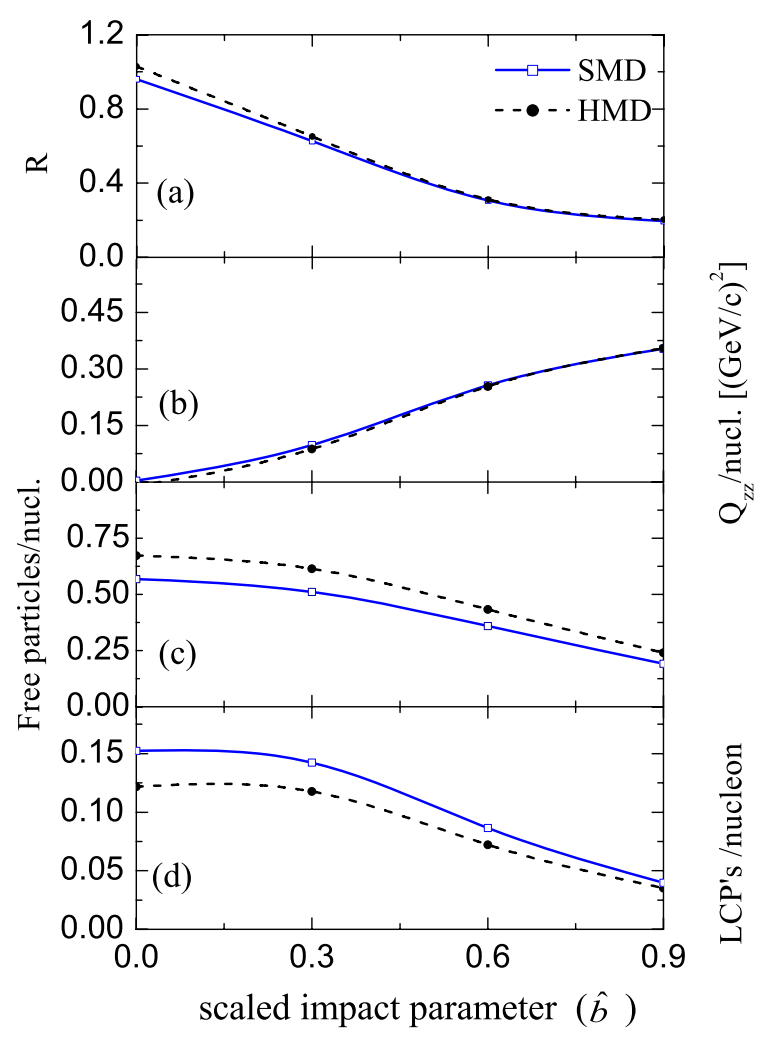

Fig. 6. Impact parameter dependence of $R(a), 1 /\left(Q_{z z} /\right.$ nucl. $)(b)$, free nucleons $(c)$, and LCPs $(d)$ with hard (HMD) and soft (SMD) equations of state with a symmetry energy of $32 \mathrm{MeV}$

creases with increase in the incident energy. Looking at the behavior of LCPs, one can say that the production of LCPs can act as an indicator of nuclear stopping.

In Fig. 6, we check the sensitivity of the nuclear stopping, as well as the multiplicity of free particles and LCPs for two different nuclear equations of states with MDI. For this purpose, the hard and soft momentum dependent equations of state with the compressibility $K=380$ and $200 \mathrm{MeV}$ are employed, respectively. As we know, the repulsive interactions are stronger with HMDE compared to SMDE at higher energies. Since the participant zone is more at lower impact parameters, one can clearly see a more stopping in the presence of HMDE. This difference keeps on decreasing, as we move from central to peripheral collisions. The production of LCPs and free particles follows the same trend as the stopping parameter $R$. This simply implies that the fragment production can act as a global indicator for the nuclear stopping.

Now we investigate the effect of isospin on the nuclear stopping. The results are displayed in Fig. 7 


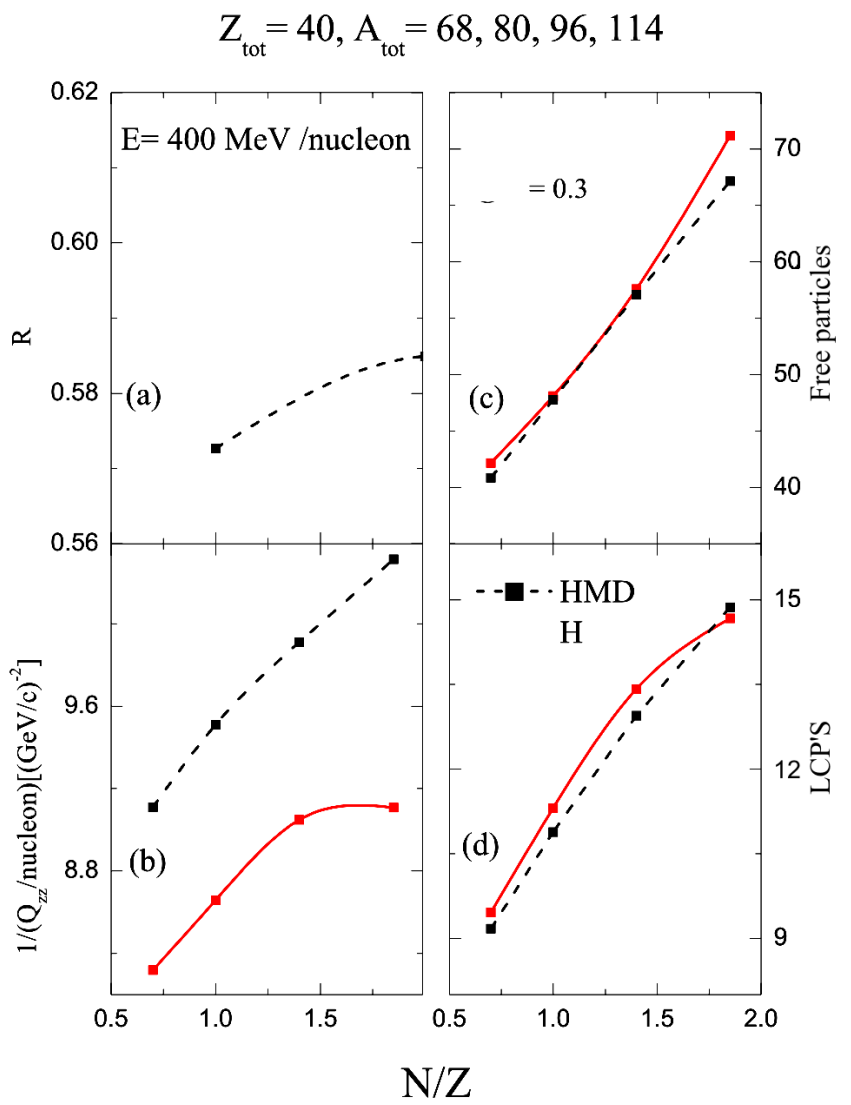

Fig. 7. $N / Z$ dependence of $R(a), 1 /\left(Q_{z z} /\right.$ nucl.) (b), free particles $(c)$, and LCPs $(d)$ in the presence of symmetry energy with HMDE and $\mathrm{HE}$

for the reactions of ${ }_{20} \mathrm{Ca}^{34}+{ }_{20} \mathrm{Ca}^{34}(N / Z=0.7)$, ${ }_{20} \mathrm{Ca}^{40}+{ }_{20} \mathrm{Ca}^{40}(N / Z=1),{ }_{20} \mathrm{Ca}^{48}+{ }_{20} \mathrm{Ca}^{48}(N / Z=$ 1.4), ${ }_{20} \mathrm{Ca}^{57}+{ }_{20} \mathrm{Ca}^{57}(N / Z=1.85)$ having the same $Z$ and different $A$ in the presence of a symmetry energy of $32 \mathrm{MeV}$ and the isospin-dependent cross-section. With increase in the $N / Z$ ratio, the number of neutrons increases, and, hence, the number of collisions increases. This, in turn, increases the stopping parameters $(R$ and $\left.1 / Q_{z z}\right)$. From the above displayed results, one may conclude that the nuclear stopping and LCPs can also be used as a tool to investigate the stopping nature of nucleons in the presence of MDI.

At last, the comparison of theoretical results with INDRA experimental data [18] is made for the reactions of $\mathrm{Au}+\mathrm{Au}$ and $\mathrm{Ni}+\mathrm{Ni}$. These simulations are carried out at $\hat{b}=0.3$ with a soft momentum-dependent equation of state (SMDE) at different energies. Our results show the overestimation of the results compared to data, because we do not have access to filters. Moreover, the

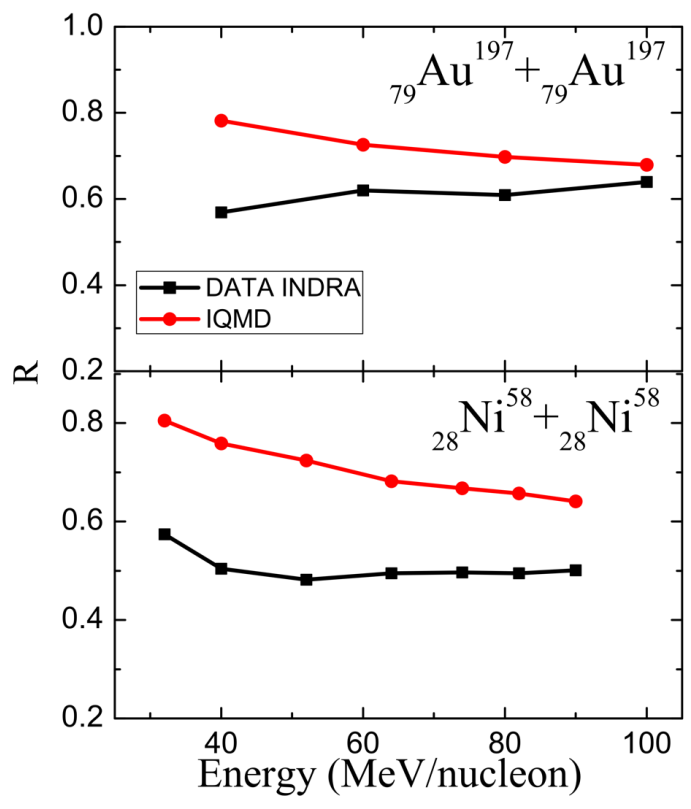

Fig. 8. Comparison of the theoretical results with experimental data for $\mathrm{Au}+\mathrm{Au}$ and $\mathrm{Ni}+\mathrm{Ni}$ at different beam energies

trend of the results is similar to experimental observations.

\section{Summary}

We studied the nuclear stopping with the momentumdependent interaction in heavy ion collisions. The analysis of the phase space, rapidity distribution, dependence of the incident energy, impact parameter, mass, and $N / Z$ shows different pictures and sensitivities toward the stopping of nuclear matter in heavy ion collisions. The nuclear stopping increases with the number of neutrons, and it decreases with increase in the incident energy and the impact parameter. In nutshell, we can say that the momentum-dependent interactions suppress the nuclear stopping.

1. J. Aichelin, Phys. Rep. 202, 233 (1991); C. Hartnack et al., Eur. Phys. J. A 1, 151 (1998); P.B. Gossiaux et al., Nucl Phys. A 619, 379 (1997); R.K. Puri et al., Phys. Rev. C 54, R28 (1996); J. Comp. Phys. 162, 245 (2000); Phys. Rev. C 57, 2744 (1998); S. Kumar et al., Phys. Rev. C 58, 3494 (1998); ibid. 58, 1618 (1998); ibid. 58, 320 (1998); ibid. 78, 064602 (2008).

2. J. Singh et.al., Phys. Rev. C 62, 044617 (2000); Y. Vermani et al., Phys. Rev. C 79, 064613 (2009); Eur. Phys. Lett 85, 62001 (2009); J. Phys G: 37, 015105 (2010); ibid. 36, 105103 (2009); J. Dhawan et al., Phys. Rev. C 74, 057901 (2006); ibid. 74, 057610 (2006). 
3. D.T. Khoa et al., Nucl Phys A 548, 102 (1992); R.K. Puri, et al., ibid. A 575, 733 (1994); E. Lehmann et al., Phys. Rev. C 51, 2113 (1995); Prog. Part. Phys. 30, 219 (1993); C. Fuchs et al., J Phys G 22, 131 (1996); Y. Vermani et al., Nucl. Phys. A 847, 243 (2010).

4. S.W. Huang et al., Prog. Nucl. Part. Physics 93, 105 (2003); Phys. Lett. B 298, 41 (1993); G. Batko et al., J. Phys. G 20, 461 (1994).

5. A.D. Sood et al., Phys. Rev. C 70, 034611 (2004); Phys. Lett. B 594, 260 (2004); Phys. Rev. C 69, 054612 (2004); ibid. 79, 064618 (2009); ibid. 73, 67602 (2006); Eur. Phys. J. A 30, 571 (2006); S. Gautum et al., J. Phys. G 37, 085102 (2010); Phys. Rev. C 83, 014603 (2011); S. Kumar, Phys. Rev. C 81, 014601 (2010); ibid. 81, 014611 (2010)

6. B. Jakobsson et al., Nucl. Phys. A 509, 195 (1990).

7. M.B. Tsang et al., Phys. Rev. Lett. 71, 1502 (1993); M.B. Tsang et al., Phys. Rev. C 49, R2271 (1994).

8. M. Begemann-Blaich et al., Phys. Rev. C 48, 610 (1993); J. Hubele et al., Phys. Rev. C 46, R1577 (1992); Z. Phys. A 340, 263 (1991); GSI report (1991).

9. W. Bauer, Phys. Rev. Lett. 61, 2534 (1998).

10. J.Y. Liu, W.J. Guo, S.J. Wang et al., Phys. Rev. Lett. 86, 975 (2001).

11. C. Gale et al., Phys. Rev. C 35, 1666 (1987).

12. J. Aichelin, A. Rosenhauer, G. Peilert, H. Stocker, and W. Greiner, Phys. Rev. Lett. 58, 1926 (1987).

13. R.K. Puri et al., Phys. Rev. C 45, 1837 (1992); ibid. 43, 315 (1991); ibid. 47, 561 (1993); Eur. Phys. J. 23, 429
(2005); J. Phys. G 18, 903 (1992); ibid. 18, 1533 (1992); Eur. Phys. J. A 3, 277 (1998); ibid. 8, 103 (2000).

14. San. Kumar and Sun. Kumar, Chin. Phys. Lett. 27, 062504 (2010).

15. T.Z. Yan, X.G. Ma, X.Z. Cai, D.Q. Feng, W. Guo, C.W. Ma, W.Q. Shen, W.D. Tian, and K. Wang, Chin. Phys. 16, 2676 (2007).

16. R.E. Renfordt et al., Phys. Rev. Lett. 53, 763 (1984).

17. W. Reisdorf et al., Phys. Rev. Lett. 92, 232301 (2004).

18. G. Lehaut et al., Phys. Rev. Lett. 104, 232701 (2010).

Received 03.02.11

ВПЛИВ ВЗАЄМОДІЙ, ЗАЛЕЖНИХ ВІД ІМПУЛЬСУ, НА ГАЛЬМУВАННЯ ЯДЕР У СИМЕТРИЧНИХ ЗІТКНЕННЯХ ВАЖКИХ ІОНІВ

M. Каур, С. Кумар

Р е з ю м е

Вивчено роль взаємодій, залежних від імпульсу (ВЗІ), у гальмуванні ядер при середніх енергіях у рамках ізоспін-залежної моделі квантової молекулярної динаміки. Обчислення виконано для енергії налітаючих частинок від 50 до $1000 \mathrm{MeB/нуклон.}$ Результати показують, що гальмування ядер чутливе до величини прицільного параметра, енергії налітаючої частинки $\mathrm{i}$ маси зіштовхувальних частинок. ВЗІ зменшують ступінь гальмування, але не впливають на народження частинок. 